\title{
DISTANCE MEASURES, INCONSISTENCY MATRICES AND ALGORITHMS FOR THE STUDY OF EPIDEMIOLOGICAL AND FINANCIAL CRISES
}

\author{
NICK JAMES
}

(Received 19 November 2021; first published online 5 January 2022)

2020 Mathematics subject classification: primary 62M10; secondary 62H12, 91G15, 92D30.

Keywords and phrases: multivariate time series, distance measures, equities, epidemiology.

This thesis introduces several new methods for multivariate time series analysis and then applies these methods to understand the behaviour of financial crises and the Covid-19 pandemic. In particular, we introduce a new semi-metric between finite sets and use it to measure distance in various settings, including between the sets of change points and turning points of time series. We also develop a new algorithmic framework to detect such change points subject to flexible parameters.

Rather than studying financial securities on an individual basis, we introduce new algorithmic frameworks to study the collective dynamics of entire asset classes and determine how these dynamics change over time. The algorithmic frameworks and distances between time series have potential applications beyond this thesis. We perform detailed analyses on equities, cryptocurrencies and international financial indices, comparing their behaviour in a range of experiments. We demonstrate substantially more homogeneity within the cryptocurrency market than the equity market, and show substantial homogeneity between the equity indices of countries during the Covid-19 pandemic.

We also study the human cost of the pandemic closely, particularly regarding the multivariate time series of cases and deaths on a country-by-country basis around the world and on a state-by-state basis in the United States. We develop several methods to determine the most suitable offset between cases and deaths, both on a country-by-country and worldwide basis. We develop methods that identify the most anomalous countries with respect to the progression of cases to deaths, classify countries and US states according to their differing wave behaviour of the disease, and reveal substantial reductions in mortality in subsequent surges. Such analysis may

Thesis submitted to the University of Sydney in April 2021, degree approved on 9 July 2021, supervisor Jennifer Chan.

(C) The Author(s), 2022. Published by Cambridge University Press on behalf of Australian Mathematical Publishing Association Inc. 
help European countries and US states alike to learn from each other's experiences and differing successes to develop the best policies to combat Covid-19 as a collective unit.

The thesis is based on seven published papers [1-7].

\section{References}

[1] N. James, 'Dynamics, behaviours, and anomaly persistence in cryptocurrencies and equities surrounding COVID-19', Phys. A 570 (2021), Article ID 125831.

[2] N. James and M. Menzies, 'Cluster-based dual evolution for multivariate time series: analyzing COVID-19', Chaos 30 (2020), Article ID 061108.

[3] N. James and M. Menzies, 'COVID-19 in the United States: trajectories and second surge behavior', Chaos 30 (2020), Article ID 091102.

[4] N. James and M. Menzies, 'Association between COVID-19 cases and international equity indices', Phys. D 417 (2021), Article ID 132809.

[5] N. James, M. Menzies, L. Azizi and J. Chan, 'Novel semi-metrics for multivariate change point analysis and anomaly detection', Phys. D 412 (2020), Article ID 132636.

[6] N. James, M. Menzies and J. Chan, 'Changes to the extreme and erratic behaviour of cryptocurrencies during COVID-19', Phys. A $\mathbf{5 6 5}$ (2021), Article ID 125581.

[7] N. James, M. Menzies and P. Radchenko, 'COVID-19 second wave mortality in Europe and the United States', Chaos 31 (2021), Article ID 031105.

NICK JAMES, School of Mathematics and Statistics,

University of Melbourne, Parkville, Victoria 3010, Australia

e-mail: njprivatemail@gmail.com 